\title{
Low power consumption lossless image compression algorithm and its application in water strider robot vision system
}

\author{
Yanming Fan ${ }^{1,}$, , Licheng $\mathrm{Wu}^{1, b^{*}}$ and ${ }^{1,}$ Xiaer $\mathrm{Li}^{1, \mathrm{c}}$ \\ ${ }^{1}$ School of Information Engineering Minzu University of China, China \\ afanyanming@muc.edu.cn, ${ }^{b}$ wulicheng@tsinghua.edu.cn, xiaer_li@163.com
}

\begin{abstract}
Keywords: miniature visual sensor, image compression, LZW, RLE
Abstract. The emergence of miniature robot makes micro vision sensing system become a hot topic in the field of scientific research. The main task of miniature visual sensor system is image acquisition and wireless transmission, but image transmission occupied the main part of the energy consumption. In order to reduce power consumption cause by wireless image -transmitting, it is necessary to compress image data. In this paper, based on MSP430 series low-power chips, we designed two modified image compression algorithm based on LZW and RLE algorithm and carry out experimental verification and comparative analysis on water strider robot vision system .
\end{abstract}

\section{Introduction}

Miniature visual sensor system applied to tiny robots becomes a hotspot in scientific research [1]. Since tiny robot is powered by limited power supply (such as button batteries) to provide energy, the visual system main features and requirement is low power consumption, that is to enable it to collect more image information under limited energy supply conditions. It can reduce the amount of data transmission greatly to compress the collected image data and reduce visual system power consumption. Image compression algorithms applied to low-power system is different from the other system image compression algorithms. Firstly, standard image compression algorithms are aimed at saving storage, not energy [2]. Secondly, because micro-processor's computing resources and storage space are limited, so the compression algorithm computational complexity should be as low as possible. Thirdly, for the image compression ratio, of course, the higher the better, but if it conflicts with energy consumption, the algorithm compression ratio will not be the main target. The most common technique for saving energy is the use of sleep mode where significant parts of the sensor's transceiver is switched off [3]. As mentioned in the paper[4][5], in most cases, the radio transceiver on board sensor nodes is the main cause of energy consumption: hence, it is important to keep the transceiver in switched off mode most of the time to save energy. One research shows that the energy consumption for executing 3000 thousands instructions is equal to the energy consumption for transmitting one thousand bit over a distance of $100 \mathrm{~m}$ by radio[6]. Therefore, how to compress image data to reduce the time of data wireless transmission becomes a key issue.

Based on LZW algorithm, paper [2] proposed a modified algorithm for wireless sensor network system called S-LZW. The main improvement is that it changes dictionary entries to 512, and there is a mini-cache of 32 entries used to store the recently used dictionary strings to improve the dictionary search speed. However, the algorithm needs more than 2KB RAM space, so it can't be applied to the platform of 2KB RAM. Paper [3] proposed an improved algorithm based on RLE, in order to improve the data compression ratio, the algorithm is a near lossless compression algorithm. In order to improve the data compression ratio, it sets $\mathrm{x}$ range, if the data is in the range, it is considered to be the same data and continues coding. The algorithm is mainly aimed at temperature data compression, so it can't be applied to image data compression. Paper [7] also proposed two improved LZW algorithm for wireless sensor networks, but they are used for continuous pulse signal, which can't be used for image data RGB565 or other formats. So in this paper, based on LZW and RLE, we have designed two kinds of modified lossless image compression algorithm and conduct comparative analysis about the two algorithms performance in a realistic WSR vision system. 


\section{Compression Algorithm Improvements}

Modified LZW algorithm. The S-LZW algorithm proposed by paper [2] can't be applied to the microprocessor RAM less than $2 \mathrm{~KB}$, in view of the microprocessor's RAM is less than $2 \mathrm{~KB}$, we improve the LZW algorithm in this paper. Paper [8] analyzes the dictionary data structure effect on the computational complexity and complexity of the LZW algorithm in detail and compares several common data structures. In this paper, we mainly improve algorithm dictionary structure, S-LZW dictionary length is 512 and it is a fixed entries, this paper change it to a shifty dictionary, that it use a linked list to add an entry dynamically. In order to improve the dictionary traversal speed, this paper designs dictionary with multi-tree data (shown as Fig.1).

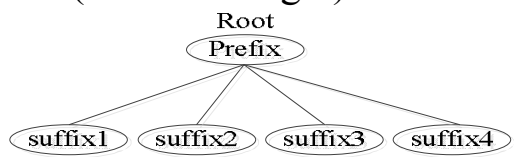

Fig.1 data structure of multi-tree

It will be a tree when string prefix is same. The dictionary establishment is implemented by applying space dynamically, if the space applied is empty, initialize the dictionary again, rebuilt dictionary. In the coding implementation process, we agree that the first 256 characters are not stored in the dictionary, which can save memory space. We named the modified algorithm as L_LZW.

Modified RLE algorithm. Take 16 bit RGB565 image data as an example, we need to encode 16 bit image data. Because of human visual characteristics, human eye resolution capability for image gray can be changed according to the gray level of image. We can derive that RGB565 format image pixels three channels can be varied from 0 to 1 from paper [9], whereby the range of the $\mathrm{R}$ and $\mathrm{B}$ channels can be 2 and $\mathrm{G}$ channel is 1 . So the process of RLE compression, if R and $\mathrm{B}$ change one gray, they can be considered the same pixel. According to the system characteristics, we improve RLE algorithm applied to PCX format, set the counter describing the repeat pixels number highest bit as 1, So there are two purposes: 1. As a marker, denote that the byte is the repeat pixels number, that is the counter; 2 . increase the counter maximum value (PCX 'counter the maximum value is 63, but this paper is 127). In addition, based on the human visual system, to judge whether (judge $\mathrm{R}$ and $\mathrm{B}$ channels) the two different pixels changes is in the range that human eye can't distinguish, if it is, we consider it is the same pixel, continue to count. We named the algorithm M_RLE.

\section{Experimental analysis}

In this paper, two kinds of image compression algorithms proposed above are carried out experiment in the micro vision sensing system and we conduct compare and analysis on the results executed by the two kinds of algorithms from three aspects: energy consumption, compression ratio and transmission time. Experiment system hardware platform is made up of a microprocessor MSP430F149 (work at the frequency of $8 \mathrm{MHz}, 2 \mathrm{~KB}$ RAM), image sensor types ov7670, wireless transmission chip $\mathrm{nRF} 24 \mathrm{~L} 01+$ (transmission rate of $1 \mathrm{Mbps}$, transmission distance is $20 \mathrm{~m}$ ), the code of the algorithm is implemented in the IAR compiler environment development, transmission packet's length is 32B.

Collect different images in different locations, the image format is RGB565 and the pixel size is $320 * 240$. Since the two algorithms are related to the data source, each image is tested for 10 times, and finally we take the average. The collected part of the compressed image is shown asFig. 2.

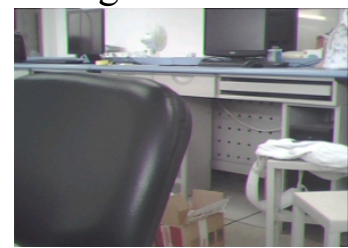

4-a

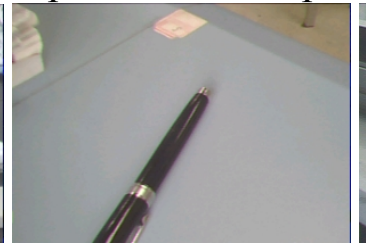

4-b

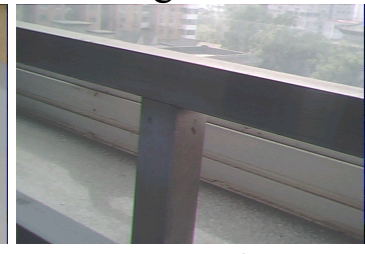

4-c

Fig.2 part of image data 
The system power consumption and the transmission time are compared at first. The experimental results are shown in Table 1.

Table 1. L_LZW and M_RLE algorithms power consumption and send time comparison table

\begin{tabular}{|c|c|c|c|c|c|c|}
\hline \multirow{2}{*}{ Image } & \multicolumn{3}{|c|}{ Time-consuming [s] } & \multicolumn{3}{c|}{ Energy consumption [mJ] } \\
\cline { 2 - 7 } & No compress & L_LZW & M_RLE & No compress & L_LZW & M_RLE \\
\hline 4-a & 8.80 & 23.50 & 6.65 & 336.16 & 897.70 & 254.30 \\
\hline 4-b & 9.10 & 19.80 & 5.45 & 347.62 & 756.36 & 208.20 \\
\hline 4-c & 9.00 & 21.70 & 6.20 & 343.80 & 828.94 & 236.84 \\
\hline
\end{tabular}

From Table 1, it can be shown that the system total energy consumption is proportional to the transmitted image time. Because the L_LZW compression algorithm is based on dictionary compression algorithm and its implementation speed is slow, it affects the image transmission time, so the energy consumption is higher than no compression and it takes long time to send image. However, the computational complexity of M_RLE algorithm is very low, and there is no need search operation, so its execution speed is fast, and the sending time is reduced, so the energy consumption is the lowest.

Then the compression ratio of the two algorithms is analyzed and its compression rate's equation is defined as (1):

$$
\text { ratio }=100 \times\left(1-\frac{\text { compressed_size }}{\text { original_size }}\right)
$$

The experiment results are shown as Fig.3.

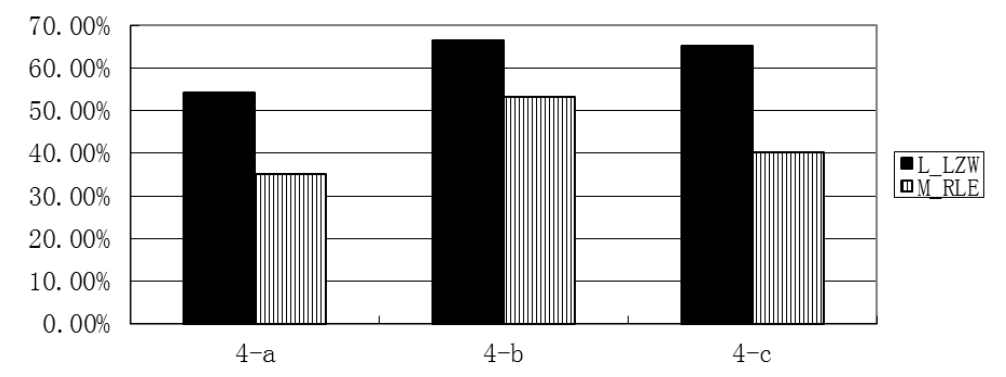

Fig.3 L_LZW and M_RLE image compression ratio chart

From Figure 5, although the compressed image is the same, the compression effect is very different. It is obvious that the compression rate of L_LZW is higher than that of M_RLE. The maximum gap between the two algorithms is $19 \%$ and the $L_{-}$LZW average compression rate is above $60 \%$. Although the compression effect of M_RLE algorithm is much less than that of L_LZW algorithm, it has a great advantage in energy consumption than LZW algorithm.

Next we compare M_RLE compression algorithm and RLE PCX image compression algorithm, the experimental results is shown in Table 2.

Table 2. M_RLE and RLE experimental results compare table

\begin{tabular}{|c|c|c|c|c|c|c|}
\hline \multirow{2}{*}{ Image } & \multicolumn{3}{|c|}{ M_RLE } & \multicolumn{3}{c|}{ RLE } \\
\cline { 2 - 7 } & Power [mJ] & Time[s] & Ratio & Power [mJ] & Time[s] & Ratio \\
\hline 4-a & 254.30 & 6.65 & $35.14 \%$ & 273.40 & 7.15 & $34.64 \%$ \\
\hline 4-b & 208.20 & 5.45 & $53.16 \%$ & 253.80 & 6.65 & $47.25 \%$ \\
\hline 4-c & 236.84 & 6.20 & $40.22 \%$ & 259.80 & 6.80 & $38.64 \%$ \\
\hline
\end{tabular}

From table 2, we can know that M_RLE and RLE algorithms compare results have less difference between, but when the acquisition image's same color pixels occupy a large proportion, the former compression effect is superior to the PCX RLE algorithms. 


\section{Conclusions}

Based on the LZW and RLE algorithm, this paper proposes two kinds of modified lossless compression algorithms for micro vision sensing systems with ultra-low power consumption chips with 2KM RAM. And conduct comparative analysis about the two algorithms performance. Results show that although RLE algorithm compression result is much less than LZW algorithm, its faster execution speed and lower energy consumption make it has great advantage in miniature low power consumption system. So we can use appropriate algorithm apply to the system according to the different system requirements.

\section{Acknowledgment}

This work was supported in part by the transition funds of the first-class university and the first-class discipline construction, the 2013 Beijing university youth talent plan No. YETP1294, the NSFC projects No. 51375504, and the Program for New Century Excellent Talents in University.

\section{References}

[1] Shen C, Chen J P, Zhen-Bo L I. The Application of Wireless Image Transmission in Micro System[J]. Microprocessors, 2007.(In Chinese)

[2] Sadler C M, Martonosi M. Data Compression Algorithms for Energy-Constrained Devices in Delay Tolerant Networks[J]. In Proc. of the ACM Conf. on Embedded Networked Sensor Systems (SenSys, 2006:265--278.

[3] Capo-Chichi E P, Guyennet H, Friedt J M. K-RLE: A New Data Compression Algorithm for Wireless Sensor Network[C]// Sensor Technologies and Applications, 2009. SENSORCOMM '09. Third International Conference on. IEEE, 2009:502 - 507.

[4] Kimura N, Latifi S. A Survey on Data Compression in Wireless Sensor Networks[C]// Information Technology: Coding and Computing, International Conference on. IEEE Computer Society, 2005:8-13.

[5] Marcelloni F, Vecchio M. A Simple Algorithm for Data Compression in Wireless Sensor Networks[J]. IEEE Communications Letters, 2008, 12(6):411 - 413.

[6] Handy M, Haase M, Timmermann D. Low Energy Adaptive Clustering Hierarchy with Deterministic Cluster-Head Selection[J]. IEEE Mwcn, 2002:368--372.

[7] Zhou Y L, Fan X P, Liu S Q, et al. Improved LZW algorithm of lossless data compression for WSN[J]. Computer Science \& Information Technology IEEE International Conference on, 2010, $4: 523-527$.

[8] Nishad P M, Chezian R M. Behavioral Study of Data Structures on Lempel Ziv Welch (LZW) Data Compression Algorithm and ITS Computational Complexity[C]// Intelligent Computing Applications (ICICA), 2014 International Conference on. IEEE, 2014:268-274.

[9] YU Tian-he,Dai Jing-min.New technology of infrared image contrast enhancement based On human visual properties[J]. Infrared and Laser Engineering 2008, 37(6):951-954. (In Chinese) 